\title{
Separation of amino acids and amines by capillary electrophoresis using poly(ethylene oxide) solution containing cetyltrimethylammonium bromide
}

\author{
Chuan-Kuo Chen ${ }^{\mathrm{a}}$, Kuntein Liu ${ }^{\mathrm{b}}$, Tai-Chia Chiuc ${ }^{\mathrm{c}, *}$, Huan-Tsung Chang ${ }^{\mathrm{a}, * *}$ \\ a Department of Chemistry, National Taiwan University, Taipei, Taiwan \\ ${ }^{\mathrm{b}}$ Radiation Application Technology Center, Institute of Nuclear Energy Research, Taoyuan, Taiwan \\ ${ }^{\mathrm{c}}$ Department of Applied Science, National Taitung University, Taitung, Taiwan
}

\section{A R T I C L E I N F O}

\section{Article history:}

Available online $\mathrm{xxx}$

\section{Keywords:}

Acetonitrile

Amines

Amino acids

Cetyltrimethylammonium bromide

Light-emitting diode-induced fluorescence detection

Micellar electrokinetic chromatography

Poly(ethylene oxide)

\begin{abstract}
A B S T R A C T
We describe simultaneous analysis of naphthalene-2,3-dicarboxaldehyde (NDA)-amino acid and amine derivatives by capillary electrophoresis in conjunction with light-emitting diode-induced fluorescence (LEDIF) detection using poly(ethylene oxide) (PEO) containing cetyltrimethylammonium bromide (CTAB). In the presence of CTAB and acetonitrile (ACN), adsorption of PEO on the capillary wall is suppressed, leading to generation of a fast and reproducible electroosmotic flow (EOF). In order to optimize separation resolution and speed, $100 \mathrm{mM}$ Tris-borate solution (pH 7.0) containing $20 \mathrm{mM} \mathrm{CTAB}$ and $25 \%$ ACN was used to fill the capillary and to prepare $1.2 \%$ PEO that entered the capillary via EOF. The analysis of 14 NDAamino acid and -amine derivatives by this approach is rapid $(<4 \mathrm{~min})$, efficient $\left((0.9-6.4) \times 10^{5}\right.$ theoretical plates), and sensitive (the LODs $(\mathrm{S} / \mathrm{N}=3)$ range from 9.5 to $50.5 \mathrm{nM})$. The RSD values $(n=5)$ of the migration times and peak heights of the analytes for the intraday analysis are less than 1.5 and $1.2 \%$, respectively. We have validated the practicality of this approach by quantitative determination of 10 amino acids and amines in a beer samples within 4 min.
\end{abstract}

(c) 2009 Published by Elsevier B.V.

\section{Introduction}

The determination of amino acids and amines in biological media is important because it is useful to understand their biological functions and to predict their possible courses of diseases. Histamine is involved in a variety of immune responses such as type I allergy [1]. Histidine (His), glutamate (Glu), valine (Val), and tyrosine (Tyr) are important markers for diagnoses of diseases, including aminoacidopathies, renal failure, and tyrosinemia type II [2-4]. Alkylamines such as methylamine have been shown to activate $\gamma \delta$ T cells and enhance immune function [5]. Norephedrine (NE) is a sympathomimetic drug, commonly used for flu, cough, cold, and rhinitis, but it may cause stroke in younger women [6] and stimulate the central nervous system. Glycine(Gly), Glu, methylamine and propylamine are important ingredients in foods such as beers, wines, tea, and milk [7-9].

\footnotetext{
* Corresponding author at: Department of Applied Science, National Taitung University, 684, Section 1, Chunghua Road, Taitung 95002,

Taiwan. Tel.: +886 $89318855 \times 3801$;

fax: +88689342539 .

** Corresponding author at: Department of Chemistry, National Taiwan University, 1, Section 4, Roosevelt Road, Taipei 106, Taiwan. Tel.: +8862 33661171; fax: +886233661171.

E-mail addresses: tcchiu@nttu.edu.tw (T.-C. Chiu), changht@ntu.edu.tw (H.-T. Chang).
}

High-performance liquid chromatography, capillary electrophoresis (CE), and gas chromatography are the most popular separation techniques for simultaneous determination of amines and amino acids $[10,11]$. CE is particularly interesting because it provides advantages of use of minimum amounts of reagents and samples, rapidity, and efficiency [12-16]. When in conjunction with laser-induced fluorescence (LIF), CE further provides high sensitivity. Because most analytes do not possess intrinsic fluorescence, derivatization of the analytes with a reagent such as naphthalene2,3-dicarboxaldehyde (NDA) is required. NDA is interesting because it allows use of a cheap and compact violet light-emitting diode (LED) as a light source. NDA reacts with primary amines in the presence of cyanide to produce cyano[ $f$ ]benzoisoindole products that fluoresce strongly at $490 \mathrm{~nm}$ after excitation at $420 \mathrm{~nm}$ [17]. CE systems in conjunction with LED-induced fluorescence (LEDIF) were employed for the analyses of amines, amino acids, and proteins [17-19]. When compared to CE-LIF systems, CE-LEDIF systems however provide lower sensitivity; usually 10 -fold lower.

Amines and amino acids can be simultaneously separated by conducting various CE modes, including micellar electrokinetic chromatography (MEKC), capillary electrochromatography (CEC), and capillary zone electrophoresis [20-22]. MEKC using sodium dodecyl sulfate is successful for the separation of amines and amino acids [20], however a highly fluorescent background in the UV region and quenching are problematic when applying fluorescence detection. Besides, we also found that repeatability is not excellent 
due to Joule heating and adsorption of the solutes in a MEKC-LIF system. CEC is powerful, but preparation of columns is tedious and usually requires an experienced researcher. The separation of amines and amino acids by CE-LIF in the presence of electroosmotic flow (EOF) under discontinuous conditions (using two different solutions to fill the capillary and buffer vials) is simple [23]. However, a long equilibrium time (about $10 \mathrm{~min}$ ) between runs, unstable baseline, and low efficiency are problematic.

In this study, we developed a simple CE-LEDIF approach for the analyses of amines and amino acids using poly(ethylene oxide) (PEO) solution containing cetyltrimethylammonium bromide (CTAB). The analytes were derivatized with NDA prior to CE-LEDIF analysis. Several important experimental parameters such as the concentrations of acetonitrile (ACN), CTAB, and PEO were investigated with respect to separation resolution and efficiency. The practicality of the proposed approach was validated by the determination of amino acids and amines in beer samples.

\section{Experimental}

\subsection{Chemicals and samples}

Amino acids and amines, including $\gamma$-aminobutyric acid (GABA), alanine (Ala), arginine (Arg), benzylamine, glutamine (Gln), Glu, Gly, His, histamine, methylamine, NE, propylamine, serine (Ser), threonine (Thr), Tyr, Val, agmatine, CTAB, and sodium cyanide $(\mathrm{NaCN})$ were obtained from Sigma (St. Louis, MO, USA). PEO $\left(M_{\mathrm{r}}\right.$ $4.0 \times 10^{6} \mathrm{~g} / \mathrm{mol}$ ) and ethanolamine were obtained from Aldrich (Milwaukee, WI, USA). Tris(hydroxymethyl)aminomethane (Tris), ACN, and methanol were purchased from J.T. Baker (Phillipsburg, NJ, USA). NDA was obtained from Tokyo Chemical Industry (Tokyo, Japan). Prior to use, NDA was dissolved in analytical grade methanol. Sodium tetraborate was obtained from Acros Organic (Geel, Belgium). Boric acid was purchased from Riedel-de Haën (Buchs, Switzerland). The stock solution of $400 \mathrm{mM}$ Tris-borate (TB) was prepared by dissolving $24.2 \mathrm{~g}$ of Tris in $500-\mathrm{mL}$ aqueous solution that was adjusted with suitable amounts of boric acid to $\mathrm{pH}$ 7.0. Unless otherwise noted, the molarity of Tris represents that for TB solution. PEO $(0.3-0.9 \mathrm{~g})$ was gradually added to each of $100 \mathrm{mM}$ TB solutions ( $\mathrm{pH} 7.0 ; 50 \mathrm{~mL}$ ) containing suitable amounts of ACN $(0-40 \%)$ and CTAB $(0.5-40 \mathrm{mM})$. During the addition of PEO, a magnetic stirring rod was used to produce a well-homogeneous suspension. After the addition was completed, the flask was sealed with paraffin to avoid evaporation of ACN. The solutions were stirred for at least $10 \mathrm{~h}$. Beer (Kirin Brewery, Tokyo, Japan) was purchased from a local store.

\subsection{Apparatus}

The basic design of the CE-LEDIF system was described previously [17]. Briefly, a high-voltage power supply (Gamma High Voltage Research, Ormond Beach, FL, USA) was used to drive electrophoresis. The entire system was placed in a black box possessing a high-voltage interlock. For safety, the high-voltage end of the separation system was placed in a laboratory-made Plexiglas box. A violet LED (InGaN; maximum output at $405 \mathrm{~nm}$ in the range 390-420 nm), obtained from Kwang-Hwa Electronic Material (Taichung, Taiwan), was used for excitation. A fused-silica capillary with $75 \mu \mathrm{m}$ I.D. and $365 \mu \mathrm{m}$ O.D. was purchased from Polymicro Technologies (Phoenix, AZ, USA). The capillary length is $40 \mathrm{~cm}$ $(30 \mathrm{~cm}$ to detector). A DV-E viscometer (Brookfield Engineering Laboratories, Middleboro, MA, USA) was employed to measure the viscosity of PEO solutions in a constant-temperature bath at $25.0 \pm 0.2{ }^{\circ} \mathrm{C}$. All measurements were performed in triplicate. A spectrofluorometer (Cary Eclipse, Varian, Palo Alto, CA, USA) was used to measure the fluorescence intensities of the NDA-amino acid and NDA-amine derivatives.

\subsection{Derivatization procedure}

The derivatization of amino acids and amines with NDA in the presence of cyanide was conducted in $1.5-\mathrm{mL}$ centrifuge tubes according to a report with slight modification [19]. Aliquots of 1.0$\mathrm{mL}$ reaction mixtures ( $\mathrm{pH} 9.3$ ) consisting of amino acids or amines (each $10.0 \mu \mathrm{M}), \mathrm{NaCN}(0.1 \mathrm{mM}), \mathrm{NDA}(0.1 \mathrm{mM})$, and sodium tetraborate $(1.0 \mathrm{mM})$ were prepared for derivatization. For the linearity test, the derivative mixture of the standard solution was sequentially diluted with water, each with a dilution factor of 2 and 5 . Each of the beer samples $(200 \mu \mathrm{L})$ that were spiked with the identified analytes $(10-500 \mu \mathrm{M})$ was mixed with water $(800 \mu \mathrm{L})$, with a dilution factor of 50 . Each of the dilute samples $(100 \mu \mathrm{L})$ was then mixed with the derivatization solution $(900 \mu \mathrm{L}, \mathrm{pH} 9.3)$ containing $0.6 \mathrm{mM}$ NDA, $1.0 \mathrm{mM}$ sodium tetraborate, and $0.6 \mathrm{mM} \mathrm{NaCN}$ in $1.5-\mathrm{mL}$ centrifuge tubes. The mixtures reacted in the dark at room temperature for $20 \mathrm{~min}$. The derivatives of standards and beer samples were further diluted for 10 -fold with de-ionized water prior to CE-LEDIF analysis.

\subsection{Electrophoretic procedure}

Prior to analysis, a $40-\mathrm{cm}$ capillary (30 $\mathrm{cm}$ in effective length) was flushed with $0.5 \mathrm{M} \mathrm{NaOH}$ for $1 \mathrm{~min}$ then was filled with TB solutions [ $100 \mathrm{mM}, \mathrm{pH} 7.0$; background electrolytes (BGEs)] containing CTAB (0.5-40 mM) and ACN (0-40\%). Hydrodynamic injection from the cathodic end was applied at $30-\mathrm{cm}$ height (the difference in the exit and entrance ends of the capillary) for $10 \mathrm{~s}$. After injection, PEO solution was introduced to the capillaries via EOF from the cathodic side. We point out that the concentrations of CTAB and ACN in the BGE were the same as those in the PEO solution in each CE-LEDIF run. The separation was conducted at $-15 \mathrm{kV}$. After each run, PEO in the capillary was flushed out and the capillary was treated with ACN for $1 \mathrm{~min}$. Followed by flushing out ACN, the capillary was equilibrated with $0.5 \mathrm{M} \mathrm{NaOH}$ at $1 \mathrm{kV}$ for $5 \mathrm{~min}$. The capillary was then filled with the BGE. This treatment was quite successful to regenerate a fast and reproducible EOF. The relative standard deviation (RSD) of EOF was less than $1.2 \%$ from run to run $(n=5)$ and less than $2.5 \%$ from day to day (five consecutive days). The shift of the baseline due to different refractive index was used to calculate the EOF mobility [19]. The LODs of amino acids and amines were estimated at the concentrations that the peak heights generated are three times the noise.

\section{Results and discussion}

\subsection{Generation of reversed EOF}

EOF direction is reversed by adsorption of CTAB on the capillary wall, in which CTAB bilayer is formed through electrostatic and hydrophobic interactions, leading to the ammonium residues toward the bulk solution [24]. In this study, CTAB was used for at least two other reasons; minimum PEO and analyte adsorption and formation of micelles as a pseudostationary phase (see section 3.2) that allow simultaneous separation of NDA-amino acid and NDA-amine derivatives. In order to generate a fast reversed EOF, a capillary was filled with a BGE that was $100 \mathrm{mM}$ TB solutions $(\mathrm{pH}$ 7.0) containing $0-40 \%$ ACN and $0.5-25 \mathrm{mM} \mathrm{CTAB} \mathrm{(Fig.} 1 \mathrm{~A}$ ). EOF was generated more reproducibly (RSD $1.2 \%$ ) at $\mathrm{pH} 7.0$ than those at $\mathrm{pH}$ values of 8.0 (1.6\%) and of 9.0 (1.8\%). At pH 7.0, the amounts of boric acid and positively charged species $\left(\mathrm{CTA}^{+}\right.$ion $)$in the solution and on the capillary wall are greater, leading to minimum PEO adsorption $[25,26]$. We point out that PEO adsorption on the capillary wall 
(A)

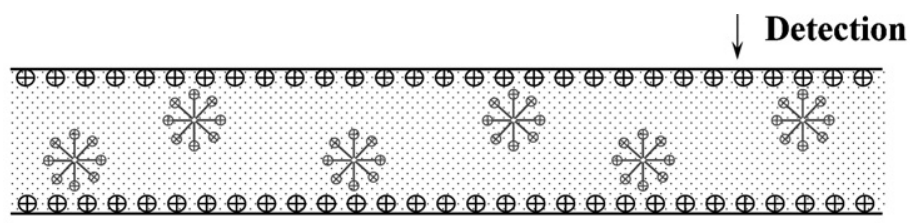

(B)
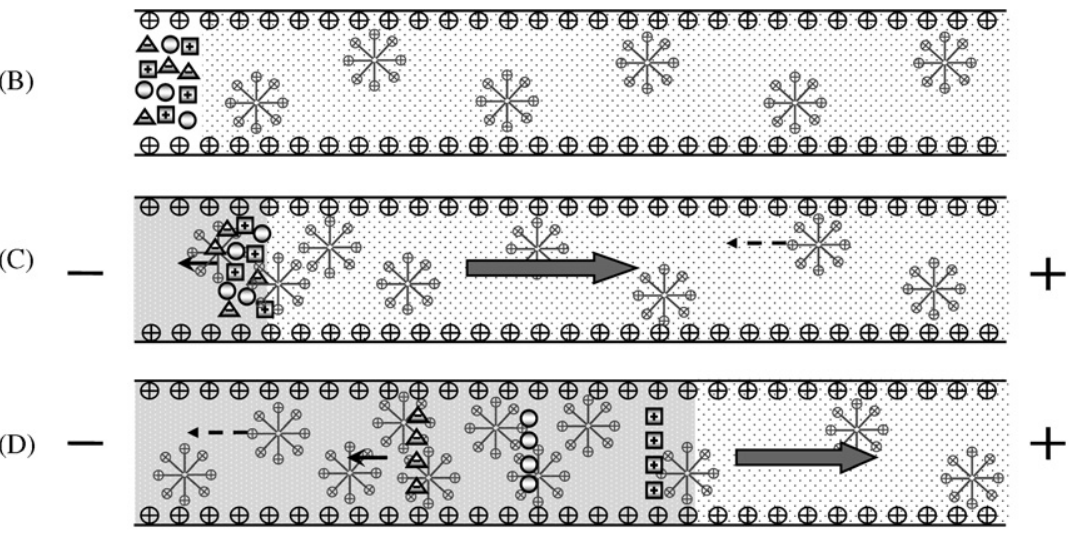

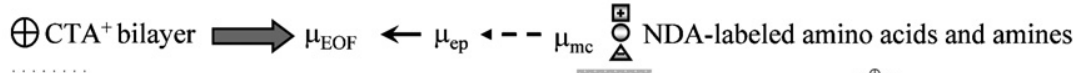

TB solution containing $\mathrm{CTAB}$ and $\mathrm{ACN} \quad \mathrm{PEO}$ solution

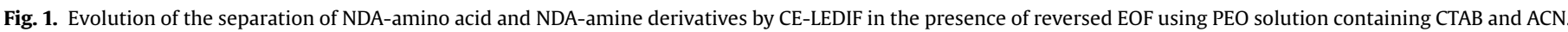

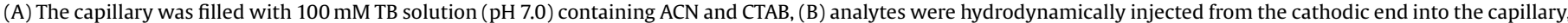

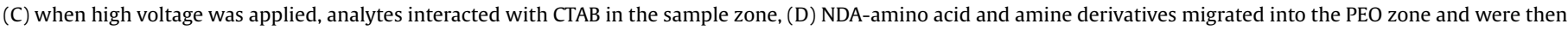
simultaneously separated. PEO solution was prepared in $100 \mathrm{mM}$ TB solution (pH 7.0) containing ACN and CTAB.

is mainly through van der Waal's force. Although a faster EOF was obtained when the TB concentration was higher than $100 \mathrm{mM}$, we experienced in problems associated with Joule hating. On the other hand, small and irreproducible EOF was generated at lower concentrations, leading to poor separation. A mixture of NDA-amino acid and NDA-amine derivatives diluted in deionized water was then hydrodynamically injected to the capillary from the cathodic end (Fig. 1B). Once high voltage was applied, PEO solution entered the capillary from the cathodic end via EOF and the derivatives were partitioned into the CTAB micelles. CTAB micelles and the positively charged CTAB complexes of NDA-amino acid and NDA-amine derivatives migrated against EOF [22]. We point out that the EOF was greater than the electrophoretic mobility of CTAB micelles and those of the analytes, and thus the analytes were all detected in the anodic side. CTAB micelles migrating from the TB zone interacted more strongly with the negatively charged NDA-amino acid derivatives than with the neutral NDA-amine derivatives (Fig. 1C). Once the negatively charged NDA-amino acid derivatives interacted with CTAB micelles, their migration towards the anodic side decreased and their electrophoretic migration direction could possibly be reversed. When NDA-amino acid and NDA-amine derivatives interacted with CTAB micelles, they migrated toward the cathode side with increased speed and then entered PEO zone. Finally, the analytes were separated and then detected by CE-LEDIF (Fig. 1D).

\subsection{Effect of $A C N, P E O$, and $C T A B$}

Organic modifiers such as ACN have long been used to enhance MEKC separations by altering solute partition coefficients and expanding the migration window $[27,28]$. When using PEO solutions, the effects of ACN on varying its viscosity and thus EOF have to be considered. We first investigated the effect of ACN on the EOF and the electrophoretic mobility of the analytes in the case of using 1.2\% PEO solutions containing 14.0 mM CTAB. As shown in Fig. 2A, the EOF mobility decreased upon increasing the ACN concentration from 0 to $40 \%$. The EOF mobility (negative; toward the anode) decreased from -7.9 to $-1.8 \times 10^{-4} \mathrm{~cm}^{2} \mathrm{~V}^{-1} \mathrm{~s}^{-1}$ when the concentration of ACN was increased from 0 to $40 \%$. Upon increasing ACN concentration, the adsorption of $\mathrm{CTA}^{+}$ions on the capillary wall decreased due to solvation, leading to decrease in EOF. In addition, an intrinsic lower dielectric constant of the mixed aqueous-ACN PEO solution results in a decline of the zeta potential. At high ACN concentrations ( $>25 \%$ ), dramatic decreases in the EOF were mainly due to a disruption or alteration of the adsorbed CTAB bilayer on the capillary wall [29]. Fig. 2B displays the changes in the electrophoretic mobility of the analytes over ACN concentration range $0-40 \%$. As shown in Fig. 2B, the positive electrophoretic mobility $\left(\mu_{\mathrm{ep}}\right)$ values (toward the cathode) for the NDA-amino acid and NDA-amine derivatives reveal that they all were partitioned in CTAB micelles. In the absence of ACN, the analytes were not resolved (comigrated) as a result of strong interactions with CTAB micelles (critical micelle concentration $0.9 \mathrm{mM}$ ) and a fast EOF [30]. Over ACN concentration range $10-30 \%$, the analytes migrated with different mobilities. When compared to the $\mu_{\mathrm{ep}}$ values of the NDA-amine derivatives, those for NDA-amino acid derivatives decreased more rapidly when the ACN concentration was increased from 25 to 35\%. Once disruption of CTAB micelles occurred, neutral NDA-amine derivatives migrated with EOF, while the negatively charged NDA-amino acid derivatives migrated with the same direction with EOF. Based on the results, we found that the optimal concentration of $A C N$ was $25 \%$ when the concentration of CTAB was $14.0 \mathrm{mM}$.

In order to verify the role that PEO plays on the separation of NDA-amino acid and NDA-amine derivatives, different concentrations $(0-1.8 \%)$ of PEO solutions were tested (Fig. 3). The entanglement threshold concentration of the PEO solution is $0.12 \%$, and thus the PEO solutions used in this study all formed networks [31]. In the absence of PEO, the peak profiles of analytes were relatively broad, mainly due to interactions of the analytes with the adsorbed CTAB bilayer on the capillary wall. With increasing PEO concentration, the resolving power and efficiency for the NDAamino acid and NDA-amine derivatives increased as a result of 
minimum analytes adsorption on the wall and decreases in diffusion of the analytes. When PEO concentration was increased from 0 to $0.6 \%$, the resolution between NDA-NE and NDA-Gly improved from 0 to 2.7. The improved resolving power was also possibly due to the interactions of the analytes with PEO molecules, mainly through hydrogen bonding and hydrophobic interactions [17,32]. However, the resolving power and efficiency become poor at PEO concentrations greater than $1.6 \%$, mainly due to the greater adsorption of PEO molecules onto the capillary wall, stronger interactions of PEO with the analytes, and significant differences in the viscosities between TB and PEO zones. Different EOF mobilities and migration times for the analytes were also due to different viscosities of various concentrations of PEO solutions [19]. The viscosity values of $0,0.1,0.6$, 1.2 , and $1.8 \%$ PEO solutions containing $14.0 \mathrm{mM} \mathrm{CTAB}$ and $25 \%$ ACN were $1.3,1.8,77,394$, and $1340 \mathrm{mPa}$ s, respectively. The EOF mobility values were $-7.4,-7.2,-6.6$, and $-6.3 \times 10^{-4} \mathrm{~cm}^{2} \mathrm{~V}^{-1} \mathrm{~s}^{-1}$ in $0,0.6$, 1.2 , and $1.8 \%$ PEO solutions containing $14.0 \mathrm{mM} \mathrm{CTAB}$ and $25 \% \mathrm{ACN}$, respectively. Fast EOF mobilities in the presence of PEO revealed that PEO adsorption on the capillary wall was not serious $[18,19]$. When using $1.2 \%$ PEO, the separation was completed within $4 \mathrm{~min}$, with the RSD values of the migration times for the analytes less than $1.5 \%(n=5)$. The plate numbers for NDA-histamine (peak 1$)$, NDA-propylamine (peak 4), and NDA-Glu (peak 8) were 1.1, 1.4, and $2.0 \times 10^{5}$ in $1.2 \%$ PEO solution, and were $4.5,2.3$, and $1.5 \times 10^{4}$ in the absence of PEO.

We also investigated the role that CTAB concentration plays on the analysis of the NDA-amino acid and NDA-amine derivatives in
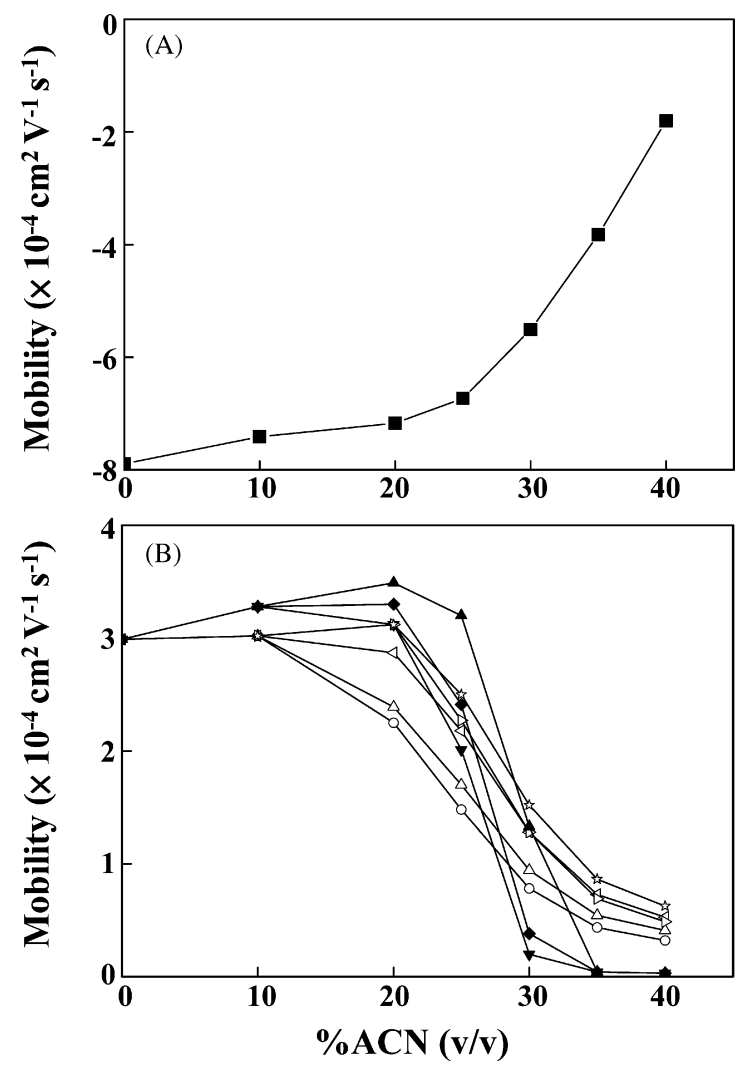

Fig. 2. Effect of $A C N$ in the BGEs on (A) EOF and (B) the electrophoretic mobilities of NDA-amino acid and NDA-amine derivatives. Capillary: $40 \mathrm{~cm}$ of total length, $30 \mathrm{~cm}$ of effective length. The BGEs were $100 \mathrm{mM}$ TB solution ( $\mathrm{pH} 7.0$ ) containing $14 \mathrm{mM}$ CTAB and ACN $[0,10,20,25,30,35$, and $40 \%(\mathrm{v} / \mathrm{v})] .1 .2 \%$ PEO solutions were prepared in the BGEs. The mixture of eight NDA-amino acid and NDA-amine derivatives $(1.0 \mu \mathrm{M}$ for each) was diluted in deionized water. Sample injection was conducted hydrodynamically by raising the capillary inlet $30-\mathrm{cm}$ height for $10 \mathrm{~s}$ and the separation was conducted at $-15 \mathrm{kV}$. Curves: $(\mathbf{\square})$, EOF; $(\bigcirc)$, histamine; $(\triangle)$, methylamine; $(\nabla)$, His; $(\triangleleft)$, propylamine; $(\triangleright)$, NE; $(\triangleleft)$, Gly; $(\succsim)$, benzylamine; and $(\boldsymbol{\Delta})$, Glu.

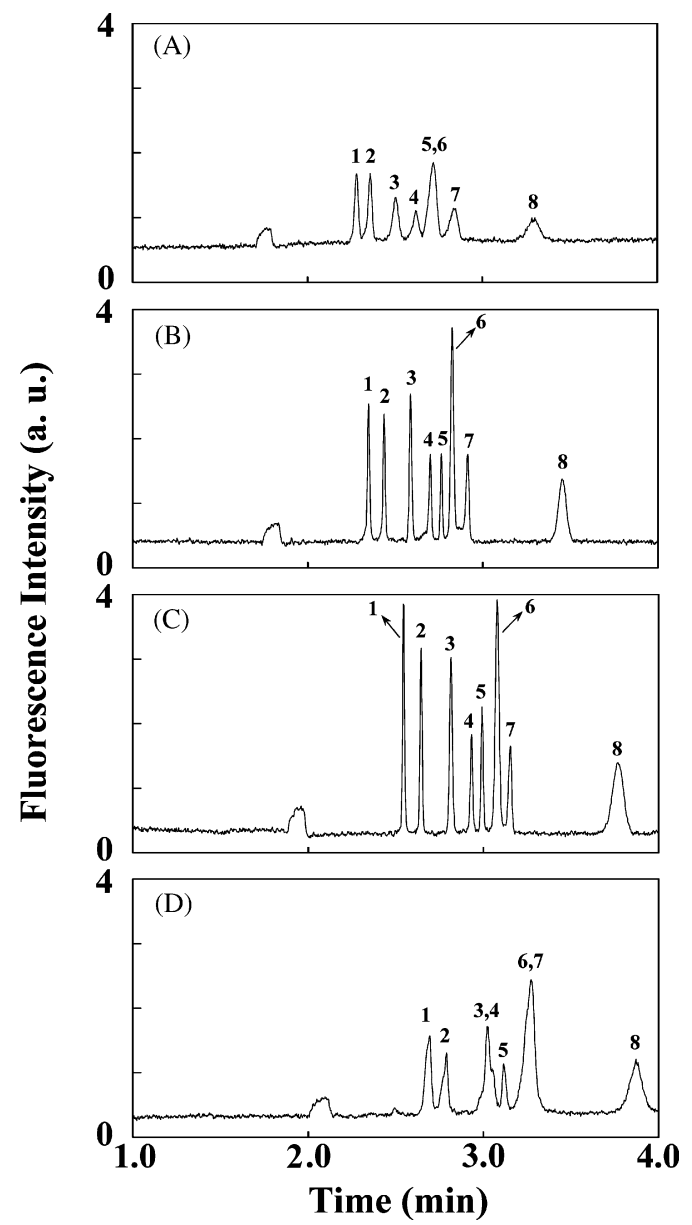

Fig. 3. Electropherograms of NDA-amino acid and NDA-amines derivatives by CELEDIF separately using (A) $0 \%$, (B) $0.6 \%$, (C) $1.2 \%$, and (D) $1.8 \%$ PEO solutions. The capillary was filled with $100 \mathrm{mM}$ TB solution ( $\mathrm{pH} 7.0$ ) containing $14.0 \mathrm{mM}$ CTAB and $25 \%$ ACN. The same TB solution was also used to prepare PEO solutions. Peak identities: 1 , histamine; 2 , methylamine; 3, His; 4, propylamine; 5 , NE; 6 , Gly; 7 , benzylamine; 8, Glu. Other conditions were the same as in Fig. $2 \mathrm{~B}$.

the case of using $1.2 \%$ PEO solutions containing $25 \% \mathrm{ACN}$. As shown in Fig. 4A, reversed EOF was obtained at the CTAB concentrations greater than $0.5 \mathrm{mM}$ [29]. Upon increasing the concentration of CTAB from 0.5 to $8.0 \mathrm{mM}$, the EOF mobility increased from -1.6 to $-6.6 \times 10^{-4} \mathrm{~cm}^{2} \mathrm{~V}^{-1} \mathrm{~s}^{-1}$, mainly due to the formation of greater amounts of $\mathrm{CTA}^{+}$bilayers on the capillary wall and decreased PEO adsorption. The plots depicted in Fig. $4 \mathrm{~B}$ reveal that the $\mu_{\mathrm{ep}}$ values of the analytes all increased upon increasing $\mathrm{CTAB}$ concentration, mainly because of increased interactions of the analytes with CTAB micelles. We note that the NDA-amino acids and NDA-amines were not separated in the absence of CTAB. Over the CTAB concentration range of $0.5-25.0 \mathrm{mM}$, the migration orders of the analytes were different and strongly dependent on CTAB concentration (interaction between CTAB and the analytes). The separation windows decreased gradually upon increasing CTAB concentration over the range $20.0-25.0 \mathrm{mM}$. In addition, peak broadening due to Joule heating became more dominant at high CTAB concentrations. The currents were 32 and $40 \mu \mathrm{A}$ when using PEO solution containing 20.0 and $25.0 \mathrm{mM} \mathrm{CTAB}$, respectively. The resolution, for instance, between NDA-NE and NDA-Gly decreased from 2.7 to 0.9 . We summarized the role of CTAB by noting that the efficiency of the analytes increased upon increasing CTAB concentration up to $20.0 \mathrm{mM}$. With respects to resolution and efficiency, the optimal concentration of CTAB was $20.0 \mathrm{mM}$. 

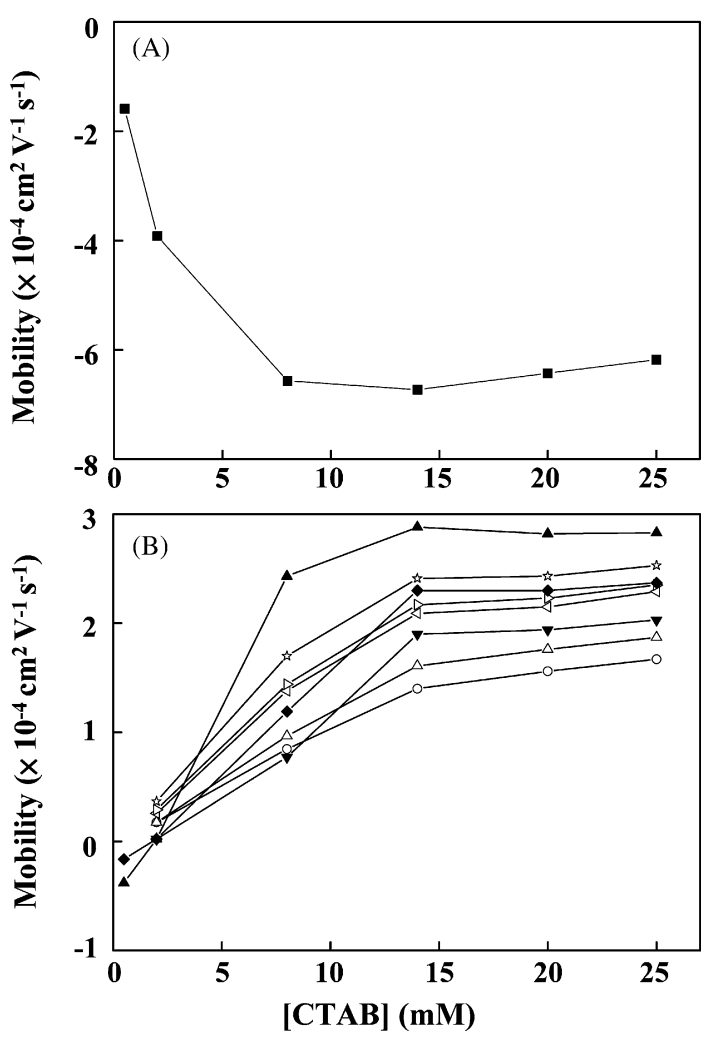

Fig. 4. Effect of CTAB concentrations in the BGEs on (A) EOF and on (B) the electrophoretic mobilities of NDA-amino acid and NDA-amine derivatives. The BGEs were $100 \mathrm{mM}$ TB solutions ( $\mathrm{pH} 7.0$ ) containing various amounts of CTAB and $25 \%$ ACN. The BGEs were used to prepare $1.2 \%$ PEO solutions. Other conditions were the same as in Fig. 3C. Curves: $(\boldsymbol{\nabla})$, EOF; $(\bigcirc)$, histamine; $(\triangle)$, methylamine; $(\boldsymbol{\nabla})$, His; $(\triangleleft)$, propylamine; $(\triangleright)$, NE; $(\diamond)$, Gly; $(\succsim)$, benzylamine; and $(\Delta)$, Glu.

\subsection{Separation of 14 amino acids and amines}

Fig. 5 exhibits the analysis of a mixture containing 14 standard NDA-amino acid and NDA-amine derivatives $(1 \mu \mathrm{M})$ by CE-LEDIF using a $40-\mathrm{cm}$ capillary under the optimal conditions. All 14 derivatives were resolved within $4 \mathrm{~min}$. The separation efficiencies for these derivatives were in the ranges of 0.9 (NDA-Glu) to $6.4 \times 10^{5}$ (NDA-Ala) theoretical plates, showing high separation efficiency. The RSD values for the migration times of the analytes were all less

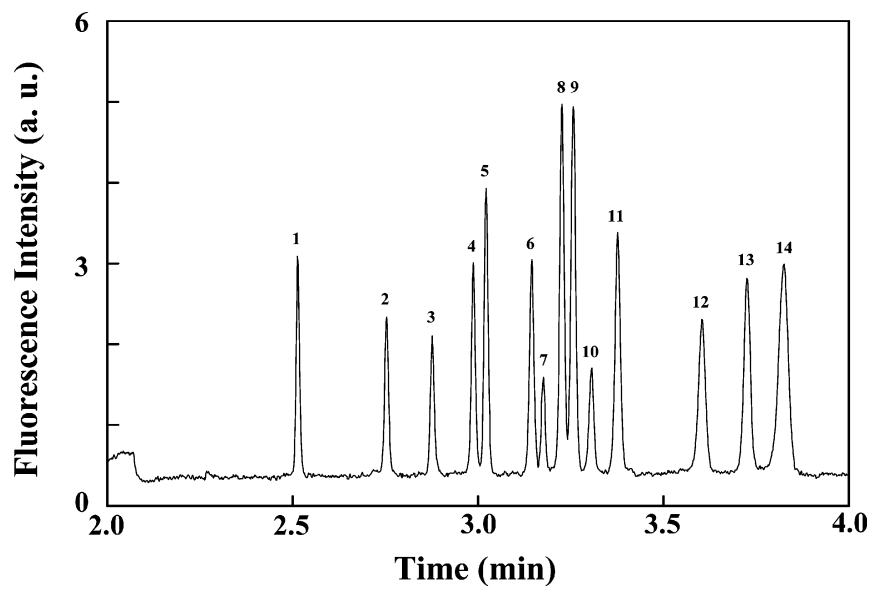

Fig. 5. Analysis of a mixture containing 14 NDA-amino acid and NDA-amine derivatives ( $1 \mu \mathrm{M}$ for each) by CE-LEDIF. Peak identities: 1, Arg; 2 , histamine; 3 , methylamine; 4, His; 5, Gln; 6, Ser; 7, propylamine; 8, GABA; 9, Gly; 10, Thr; 11, Ala; 12, Tyr; 13, Val; 14, Glu. Other conditions were the same as in Fig. 4B.

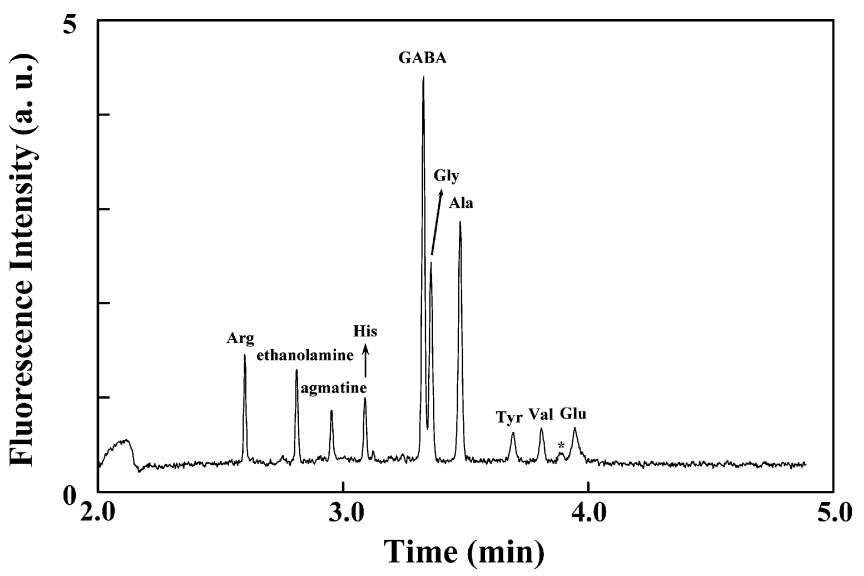

Fig. 6. Electropherogram of a 500-fold diluted beer sample by CE-LEDIF. Other conditions were the same as in Fig. 5.

than $1.5 \%(n=5)$. To test the quantitative capability of this approach, different concentrations $(0.1-10.0 \mu \mathrm{M})$ of the NDA-amino acid and NDA-amine derivatives were subjected to CE-LEDIF analyses. The linearity $\left(R^{2}>0.98\right)$ for each of the NDA-amino acid and NDA-amine derivatives was good, showing the great potential of this approach for the simultaneous analysis of amino acids and amines. Based on the electropherogram of NDA-amino acid and NDA-amine derivatives (100 nM), we estimated the LODs at $\mathrm{S} / \mathrm{N}=3$ for Arg, histamine, methylamine, His, Gln, Ser, propylamine, GABA, Gly, Thr, Ala, Tyr, Val, and Glu to be 13.6, 13.2, 14.8, 17.7, 14.8, 16.3, 40.1, 9.5, 10.1, 50.5, $16.6,25.5,20.3$, and $18.0 \mathrm{nM}$, respectively.

\subsection{Analysis of a beer sample}

To test the practicality of the proposed approach, the concentrations of amino acids and amines in beer samples were determined. Fig. 6 exhibits a representative electropherogram of separating a diluted beer sample under the optimal separation conditions. The RSD values (five repetitive runs) of the migration times for the identified amino acids were less than 1.5\%. The day-to-day (five consecutive days) and capillary-to-capillary (five different capillaries) RSD values were less than 2.5 and $2.1 \%$, respectively. Because CE-LEDIF was applied, fewer peaks were detected than those by applying CE with UV-vis absorption detection. The differences in the migration times of the analytes in Figs. 5 and 6 show a matrix effect of the beer sample. By using a standard addition method (peak height) $\left(R^{2}>0.98\right)$, the concentrations of Arg, ethanolamine, agmatine, His, GABA, Gly, Ala, Tyr, Val, and Glu in the beer sample were estimated to be $174.1 \pm 5.7,103.2 \pm 4.2,87.4 \pm 3.8,92.3 \pm 3.3$, $428.8 \pm 9.7,258.6 \pm 6.8,415.3 \pm 8.0,136.2 \pm 3.5,109.2 \pm 3.1$, and $89.8 \pm 2.7 \mu \mathrm{M}$, respectively (five repetitive runs). The values were in good agreement with the literatures [7,33-35]. Since NDA only forms fluorescent derivatives with solutes possessing primary amino groups, the unidentified peak marked as * is mainly corresponding to compound(s) having primary amino groups.

\section{Conclusions}

In this study, 14 NDA-amino acid and amine derivatives were successfully separated by CE-LEDIF. Buffer composition, the concentrations of PEO and CTAB, and the amount of ACN were all proved to play some roles in the analysis of these analytes. To the best of our knowledge, this is the first example for the separation of amino acids and amines in PEO solution containing ACN and CTAB. We have found that CTAB is effective to suppress PEO adsorption, ensuring generation of fast and reproducible EOF. Using 
our new capillary treating strategy prevents tedious washing and equilibration processes when using polymer solutions. Under the optimum conditions, the separation of 14 NDA-amino acid and amine derivatives was achieved within 4 min by CE-LEDIF. The present CE approach provides advantages of simplicity, rapidity, high efficiency, and high sensitivity.

\section{Acknowledgements}

This work was supported by the National Science Council of Taiwan under contract numbers NSC 95-2113-M-002-026-MY3 and NSC 97-2113-M-143-001-MY2.

\section{References}

[1] A.D. Crockard, M. Ennis, Clin. Exp. Allergy 31 (2001) 345.

[2] M.T. Veledeo, M. de Frutos, J.C. Diez-Masa, Electrophoresis 27 (2006) 3101.

[3] A. Tom, K.S. Nair, J. Nutr. 136 (2006) 324S.

[4] M. Valikhani, M. Akhyani, A.K.Jafari, M. Barzegari, S. Toosi,J. Eur. Acad. Dermatol. Venereol 20 (2006) 591.

[5] S.S. Percival, J.F. Bukowski, J. Milner, J. Nutr. 138 (2008) 1.

[6] A. Figueras, J.-R. Laporte, Drug Safety 25 (2002) 689

[7] D. Kutlán, I. Molnár-Perl, J. Chromatogr. A 987 (2003) 311.

[8] M.-M. Hsieh, S.-M. Chen, Talanta 73 (2007) 326.

[9] V. García-Cañas, A. Cifuentes, Electrophoresis 29 (2008) 294.

[10] T.-C. Chiu, Y.-W. Lin, Y.-F. Huang, H.-T. Chang, Electrophoresis 27 (2006) 4792.

[11] A. Önal, Food Chem. 103 (2007) 1475.
[12] S.L. Simpson Jr., J.P. Quirino, S. Terabe, J. Chromatogr. A 1184 (2008) 504.

[13] S. Terabe, Chem. Rec. 8 (2008) 291.

[14] K. Sueyoshi, F. Kitagawa, K. Otsuka, Anal. Chem. 80 (2008) 1255

[15] F. Kitagawa, T. Tsuneka, Y. Akimoto, K. Sueyoshi, K. Uchiyama, A. Hattori, K. Otsuka, J. Chromatogr. A 1106 (2006) 36.

[16] T. Ikegami, K. Tomomatsu, H. Takubo, K. Horie, N. Tanaka, J. Chromatogr. A 1184 (2008) 474.

[17] P.-L. Chang, T.-C. Chiu, H.-T. Chang, Electrophoresis 27 (2006) 1922.

[18] T.-C. Chiu, H.-T. Chang, J. Chromatogr. A 1146 (2007) 118.

[19] T.-C. Chiu, W.-C. Tu, H.-T. Chang, Electrophoresis 29 (2008) 433.

[20] L. Zhou, S. Wang, K. Tian, Y. Dong, Z. Hu, J. Sep. Sci. 30 (2007) 110.

[21] Z. Chen, T. Hobo, Electrophoresis 22 (2001) 3339.

[22] L.-Y. Zhang, M.-X. Sun, J. Chromatogr. A 1040 (2004) 133.

[23] M.-M. Hsieh, H.-T. Chang, Electrophoresis 26 (2005) 187.

[24] C.A. Lucy, R.S. Underhill, Anal. Chem. 68 (1996) 300.

[25] W.-L. Tseng, M.-M. Hsieh, S.-J. Wang, H.-T. Chang, J. Chromatogr. A 894 (2000) 219.

[26] W.-L. Tseng, H.-T. Chang, Anal. Chem. 72 (2000) 4805

[27] C.A. Silva, E.A. Pereira, G.A. Micke, J.P.S. Farah, M.F.M. Tavares, Electrophoresis 28 (2007) 3722.

[28] M. Silva, Electrophoresis 28 (2007) 174

[29] A.G. Diress, C.A. Lucy, J. Chromatogr. A 1027 (2004) 185.

[30] J.-F. Liu, W.A. Ducker, J. Phys. Chem. B 103 (1999) 8558.

[31] M.-M. Hsieh, T.-C. Chiu, W.-L. Tseng, H.-T. Chang, Curr. Anal. Chem. 2 (2006) 17.

[32] M.-M. Hsieh, C.-E. Hsu, W.-L. Tseng, H.-T. Chang, Electrophoresis 23 (2002) 1633.

[33] T. Erbe, H. Brückner, J. Chromatogr. A 881 (2000) 81.

[34] S. Casado-Terrones, S. Cortacero-Ramírez, A. Carrasco-Pancorbo, A. SeguraCarretero, A. Fernández-Gutiérrez, Anal. Bioanal. Chem. 386 (2006) 1835.

[35] Q.-S. Qu, X.-Q. Tang, C.-Y. Wang, G.-J. Yang, X.-Y. Hu, X. Lu, Y. Liu, S.-C. Li, C. Yan, Anal. Chim. Acta 572 (2006) 212 